\title{
Streaming Currents in a Single Nanofluidic Channel
}

\author{
Frank H. J. van der Heyden, Derek Stein, and Cees Dekker \\ Kavli Institute of Nanoscience, Delft University of Technology, Lorentzweg 1, 2628 CJ Delft, The Netherlands
}

(Received 4 May 2005; published 8 September 2005)

\begin{abstract}
We report measurements of the streaming current, an electrical current generated by a pressure-driven liquid flow, in individual rectangular silica nanochannels down to $70 \mathrm{~nm}$ in height. The streaming current is observed to be proportional to the pressure gradient and increases with the channel height. As a function of salt concentration, it is approximately constant below $\sim 10 \mathrm{mM}$, whereas it strongly decreases at higher salt. Changing the sign of the surface charge is found to reverse the streaming current. The data are best modeled using a nonlinear Poisson-Boltzmann theory that includes the salt-dependent hydration state of the silica surface.
\end{abstract}

PACS numbers: $82.65 .+r, 66.90 .+r$

Nanofluidics is an emerging field of interest because of its potential to study and manipulate fluids, nanoparticles, and molecules in lab-on-a-chip devices [1-5]. Electrokinetic phenomena such as electro-osmotic flow and streaming currents [6-8], known to be induced by the charged walls of a microchannel, will become increasingly important in the nano regime because of the larger surface to volume ratio [9]. Moreover, new physical effects may occur as the counterion distributions of electrical double layers at opposite surfaces of a nanochannel start to overlap [10]. So far, the effects of surface charge and potential on electrokinetic fluid phenomena in confined nanostructures have been largely unexplored.

Streaming currents provide a useful technique for studying the electric properties of the solid-liquid interface as they result from the transport of counterions in the electric double layer by an applied pressure gradient [Fig. 1(d)]. It has also been suggested that streaming currents in fluidic channels may provide a simple and effective means of converting hydrostatic pressure differences into electrical energy [11], and that this process becomes particularly efficient in the nano regime where double layers overlap [10]. Streaming currents have been primarily studied on systems involving many channels in parallel, using materials such as sandstone cores [12], porous glass [11], and columns packed with latex beads [13]. While studies have appeared on single channels in the micron range $[6,14,15]$, nanofabrication now permits us to investigate streaming currents in well-defined, nanoscale geometries where double layers may overlap.

In this Letter, we report measurements of streaming currents in individual rectangular silica nanochannels as a function of pressure, channel height, and salt concentration. The data are compared with different models for the electrostatic properties of the surface: constant charge, constant potential, and a model that incorporates the chemical nature of the silica surface.

Nanofluidic channels were fabricated following a silicate bonding procedure similar to Stein et al. [9]. Channels $50 \mu \mathrm{m}$ wide and $4.5 \mathrm{~mm}$ long were patterned between
$1.5 \times 1.5 \mathrm{~mm}^{2}$ reservoirs on a fused silica substrate [Fig. 1(a)] by electron-beam lithography. The channels were plasma etched to a depth, $h$, ranging from $1147 \mathrm{~nm}$ down to $70 \mathrm{~nm}$. After a thorough cleaning procedure that ended with a $1 \mathrm{~min}$ dip in $0.5 \%$ aqueous HF solution, the channels were sealed by first spinning a $20 \mathrm{~nm}$ layer of sodium silicate from $2 \%$ aqueous solution onto a flat fused silica chip and then pressing the silicate-coated surface to the patterned channel surface, and finally curing the device at $100^{\circ} \mathrm{C}$ for $2 \mathrm{~h}$. The channels were connected to a Plexiglas holder using silicone $\mathrm{O}$ rings to make a watertight seal. Aqueous solutions, when inserted on one side of the holder, were drawn by capillary force into the nanochannels. Peek tubing connected one side of the channel to a pressurized chamber, while the opposite side was left open to atmosphere, as illustrated in Fig. 1(b). Electrical connections were established by inserting a $\mathrm{Ag} / \mathrm{AgCl}$ (a)

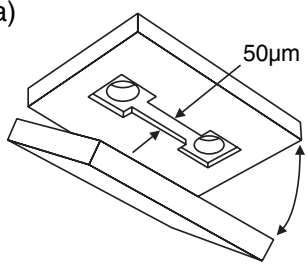

(c)

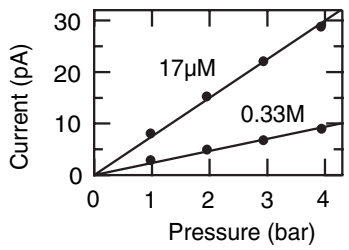

(b)

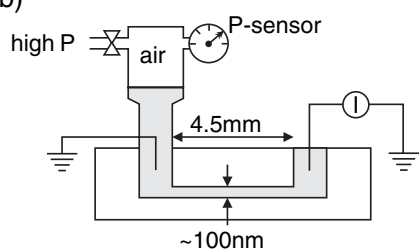

(d)



FIG. 1. (a) Nanochannels are fabricated by bonding two pieces of fused silica. The top piece contains an etched nanochannel and two $1 \mathrm{~mm}$ diameter holes for fluid connections. (b) Side view of the nanochannel. (c) Streaming current as a function of pressure for a $140 \mathrm{~nm}$ high channel at low- and high-salt concentrations. Lines are linear fits. (d) Schematic illustration of the origin of the streaming current. 
electrode through a $\mathrm{T}$ junction on the pressurized side, and a plain $\mathrm{Ag} / \mathrm{AgCl}$ electrode wire into the liquid on the opposite side. The applied pressure was controlled to values of up to 4 bar using a Festo-LRP-1/4-4 pressure regulator and was continuously monitored using a calibrated Honeywell 24PC pressure sensor. A correction was made for the small pressure drop of about $2 \%$ across the channel entrance and exit, which was simulated using fluid dynamics modeling software. Potassium chloride $(\mathrm{KCl})$ solutions ranging from $5.6 \mu M$ to $1 M$ were made by the serial dilution of a $1 M \mathrm{KCl}, 10 \mathrm{~m} M$ TRIS solution $(p \mathrm{H}=8.0)$ with distilled deionized water $(18 \mathrm{M} \Omega \mathrm{cm})$. A pressure of 4 bar was applied to remove any air bubbles from the channel and to achieve a stable streaming current. Approximately $5 \mathrm{~h}$ of stabilization time were allowed after initially filling the channel, and about $1 \mathrm{~h}$ for each subsequent exchange of salt solution. After stabilizing, streaming currents were measured while applying pressures that were varied from 1 to 4 bar in $80 \mathrm{~s}$ steps.

The dependence of the measured streaming current, $I_{\mathrm{str}}$, on the applied pressure across a $140 \mathrm{~nm}$ channel is plotted in Fig. 1(c) for salt concentrations of $17 \mu M$ and $0.33 M$. Clearly $I_{\text {str }}$ scales linearly with pressure for both salt concentrations. We define a streaming conductance, $S_{\text {str }}$, as the streaming current per unit applied pressure. $S_{\text {str }}$ is clearly higher for the low $\mathrm{KCl}$ concentration, as can be seen from the slopes in Fig. 1(c). This contrasts the electrical conductance in nanochannels, which decreases at low salt [9].

Figure 2 shows $S_{\text {str }}$ as a function of $\mathrm{KCl}$ concentration, $c$, for a $140 \mathrm{~nm}$ channel. Data points are averaged over two data sets, and the error bars indicate the average standard deviation for all points. The data show that $S_{\text {str }}$ is approximately constant for $c$ below $10 \mathrm{mM}$, and decreases at higher $c$ where it has dropped by an order of magnitude at $c=1 M \mathrm{KCl}$. The same trend is observed for different channel heights as shown in Fig. 3, which shows $S_{\text {str }}$ versus

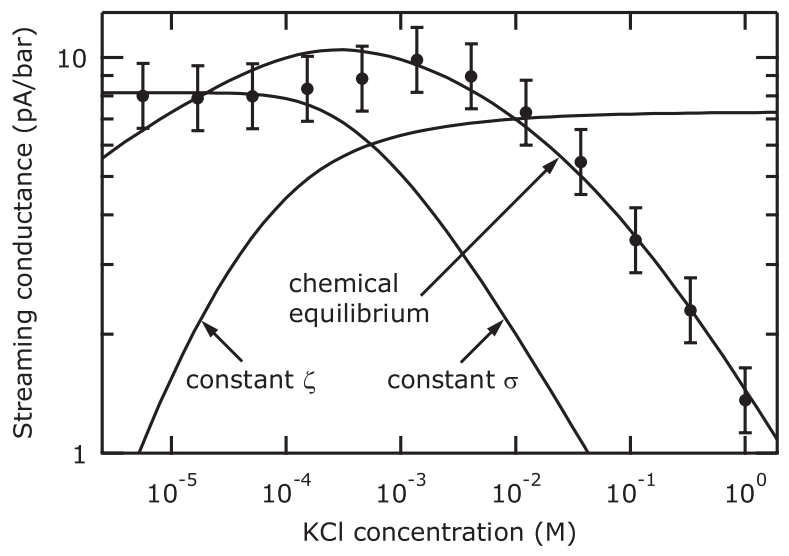

FIG. 2. Streaming conductance as a function of $\mathrm{KCl}$ concentration for a $140 \mathrm{~nm}$ high channel. Lines show model curves of constant surface charge, constant zeta potential, and the chemical equilibrium model, treated here in this Letter. $c$ for channels ranging from 70 to $1147 \mathrm{~nm}$. The open symbols represent data points at very high molarity, where values for $S_{\text {str }}$ appear to be depressed. We attribute this to deviations from the mean-field model or to effects of surface roughness, as the double layer is very compact here. Higher channels are found to yield a larger streaming current. For all channel heights, $S_{\text {str }}$ reaches a constant value in the low-salt regime, and decreases at high salt. The low-salt plateau extends to higher $c$ for smaller channels.

We begin modeling these data by equating the streaming current $I_{\text {str }}$ to the product of the charge density, $\rho(x)$, and the local speed of the fluid, $u(x)$, integrated over the cross section of the channel:

$$
I_{\mathrm{str}}=w \int_{-h / 2}^{h / 2} \rho(x) u(x) d x,
$$

where $x$ is the height from the channel midplane, $w$ is the channel width, and $h$ is the channel height. We ignore sidewall effects because $w \gg h$, and treat the flow and charge density profiles as uniform over the width of the channel, as in an infinite parallel-plate geometry. The velocity distribution is described by a Poiseuille flow subject to the no-slip boundary condition at the walls: $u(x)=$ $-\frac{\Delta P}{8 L \eta}\left(h^{2}-4 x^{2}\right)$, where $L$ is the length of the channel, $\Delta P$ the pressure difference, and $\eta$ the viscosity. We describe $\rho(x)$ using nonlinear Poisson-Boltzmann theory, starting with the Poisson-Boltzmann equation for the electrostatic potential distribution $\psi(x)$ :

$$
\frac{d^{2} \psi(x)}{d x^{2}}=\frac{k T \kappa^{2}}{e} \sinh \left(\frac{e \psi(x)}{k T}\right)
$$

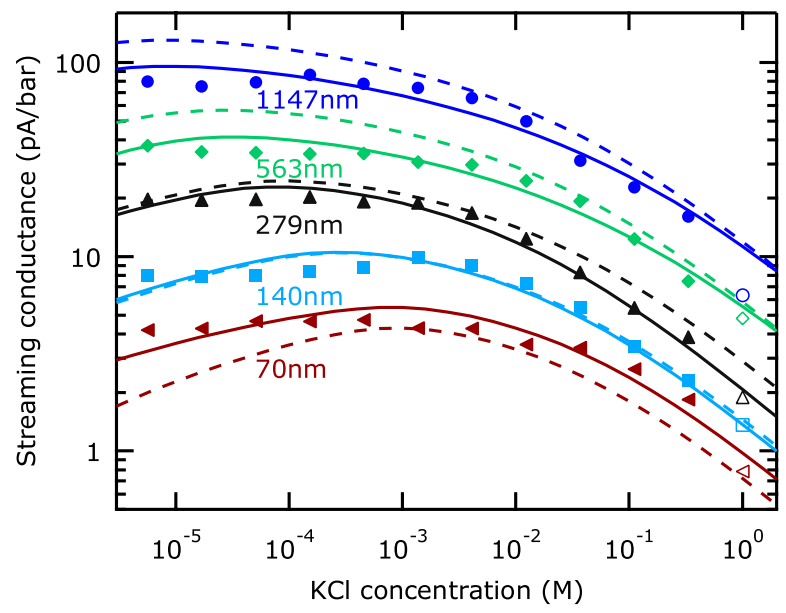

FIG. 3 (color online). Streaming conductance as a function of $\mathrm{KCl}$ concentration for five different channel heights. Data points are averages of two data sets. The dashed lines represent the chemical equilibrium model for a single set of parameters, whereas the solid lines represent the same model when the parameters $C$ and $p \mathrm{~K}$ are different for each curve. 
where $e$ is the electron charge, $k$ is the Boltzmann constant, $T$ is the temperature, and $\kappa^{-1}$ is the Debye screening length, defined by $\kappa^{2}=\frac{2 e^{2} n}{\epsilon \epsilon_{0} k T}$. Here, $\epsilon_{0}$ is the permittivity of the vacuum, $\epsilon$ is the dielectric constant of water, and $n$ is the number density of the monovalent ions, proportional to $c$. The analytical solution for $\psi(x)$ is given by [16]

$$
\psi(x)=\psi_{0}+\frac{2 k T}{e} \ln (\operatorname{JacCD}(z \mid m)),
$$

where $\psi_{0}$ is the potential in the center of the channel, and $\operatorname{JacCD}(z \mid m)$ is the Jacobi elliptical function with argument $z=\frac{\kappa x}{2} e^{-e \psi_{0} / 2 k T}$ and parameter $m=e^{2 e \psi_{0} / k T}$. The electric field, $E(x)$, and the charge density, $\rho(x)$, are obtained from $\psi(x)$ through $E(x)=-\frac{d \psi(x)}{d x}$ and Poisson's equation, $\rho(x)=-\epsilon \epsilon_{0} \frac{d^{2} \psi(x)}{d x^{2}}$.

We now compare the predictions of this model for the streaming current, assuming different boundary conditions for the electrical surface properties: constant surface charge $\sigma$, constant zeta potential $\zeta$, or a chemically variable $\sigma$. Constant $\sigma$ is imposed using Gauss' law: $\sigma=$ $\pm \epsilon \epsilon_{0} E\left(x=\mp \frac{h}{2}\right)$, and constant $\zeta$ by specifying the potential at the no-slip plane: $\zeta=\psi\left(x= \pm \frac{h}{2}\right)$.

For any given $h$ and $\kappa$, Eq. (3) is solved using either of these boundary conditions to give a value for $\psi_{0}$, and thereby $\psi(x)$ and $\rho(x)$ are solved through Eq. (3) and Poisson's equation. In the third model, we account for the chemical reactivity of the surface by allowing $\sigma$ to vary due to proton transfer through the following equilibrium [17]:

$$
\mathrm{SiOH} \rightleftharpoons \mathrm{SiO}^{-}+\mathrm{H}^{+} .
$$

Behrens and Grier [16] developed a model for the diffuse layer potential, which we take to be $\zeta$, by describing the dissociation of $\mathrm{SiOH}$ groups using equilibrium constant, $p \mathrm{~K}$, and the compact Stern layer using capacitance, $C$, yielding the following relation for $\zeta$ :

$$
\zeta(\sigma)=\frac{k T}{e} \ln \left(\frac{-\sigma}{e \Gamma+\sigma}\right)+\frac{\ln (10)}{e / k T}(p \mathrm{~K}-p \mathrm{H})-\frac{\sigma}{C},
$$

where $\Gamma$ is the surface density of chargeable sites. A second relation between $\zeta$ and $\sigma$ derives from the charge and potential distribution in the diffuse layer. For the case where $h \gg \kappa^{-1}$, no double layer overlap occurs, and the surface charge density is approximated using the Grahame equation:

$$
\sigma(\zeta)=\frac{2 \epsilon \epsilon_{0} k T \kappa}{e} \sinh \left(\frac{e \zeta}{2 k T}\right) .
$$

Solving Eqs. (5) and (6) yields a value for the surface charge $\sigma$, or potential $\zeta$. When double layer overlap does occur, no analytical solution is available. In this case $\psi(x)$, and thereby $\zeta$, were solved for a set of $\sigma$ values as a boundary condition. $\zeta$ was plotted as a function of $\sigma$, and its intersection point with the chemical equilibrium relation, Eq. (5), was determined. The intersection point yields self-consistent solutions for $\sigma$ and $\zeta$ that both depend on $\kappa$ and $h$. The $\sigma$ thus obtained was used as a boundary condition to find $\rho(x)$ and $S_{\text {str }}$. The model predicts that $\sigma$ increases with the salt concentration and that its dependence on $h$ is relatively unimportant for the channel sizes and salt concentrations that we have used: at the lowest salt concentration, 5.6 $\mu M$, and the smallest channel, $70 \mathrm{~nm}, \sigma$ is predicted to be reduced by just $1.3 \%$ relative to the largest channel of $1147 \mathrm{~nm}$.

We now compare these three models for the streaming current in silica nanochannels. The predictions for $S_{\text {str }}$ in a nanochannel as a function of $c$ are plotted in Fig. 2 for $\zeta=$ $-60 \mathrm{mV}, \sigma=-4 \mathrm{mC} / \mathrm{m}^{2}$, and the chemical equilibrium boundary conditions with parameters $\Gamma=8 \mathrm{~nm}^{-2}, p \mathrm{~K}=$ $7.9, p \mathrm{H}=8$, and $C=0.3 \mathrm{Fm}^{-2}$. Note that the latter values are not free fit parameters, but were adopted from literature [18]. From Fig. 2, it is clear that the constant- $\zeta$ line fails to describe our data, as it is constant at high salt and drops when $c$ is decreased below $100 \mu M$. The constant- $\sigma$ model, which previously described the salt dependence of the electrical conductance successfully [9], does a somewhat better job: it yields a plateau at low salt and drops off at high salt. However, the current decrease sets in at significantly lower $c(\sim 1 \mathrm{mM})$ than observed $(\sim 10 \mathrm{mM})$, leading to significant deviations between model and data. The chemical equilibrium model gives the best fit over the full range of $c$, predicting a roughly constant low-salt plateau, and the correct highsalt decay.

In Fig. 3, the dashed lines represent predictions of the chemical equilibrium model for the same single set of parameters that are applied to all curves [18]. This model describes the data reasonably well over 5 orders of magnitude in salt concentration and a factor of 16 in channel height, using a single set of material parameters. Since the properties of silica can vary between channels, we may fit the parameters $p \mathrm{~K}$ and $C$ for each curve [19] to obtain a very good description of $S_{\text {str }}$ for all channels, presented using solid lines.

We also demonstrate the sensitivity of the streaming current in nanochannels to the sign of the surface charge by inverting the surface charge. For this purpose, we coated a fused silica nanochannel with a layer of polylysine by incubating the channel in $0.01 \% 70-150 \mathrm{kDa}$ polylysine solution for $12 \mathrm{~h}$, which is expected to render the surface positively charged. Indeed, Fig. 4 shows that $I_{\text {str }}$ in the direction of the liquid flow is negative for polylysinecoated channels, which indicates that the double layers are populated with negative ions, whereas the untreated silica surface generates a positive current, indicating positive ions in the double layer [20].

Finally, we comment on the proposal that the streamingcurrent effect can be used to convert hydrostatic energy into electrical energy [11]. Using the exact Poisson- 




FIG. 4. Effect of the sign of the surface charge on the streaming current. The streaming current is shown as a function of pressure for a $279 \mathrm{~nm}$ high nanochannel at low- and high-salt concentration. Data are shown for a negatively charged silica surface, and for a polylysine-coated surface that is positively charged. Lines indicate linear fits.

Boltzmann solution for the charge distribution, combined with the chemical equilibrium boundary condition for the silica surface using a single set of material parameters [18], we find that a maximum energy conversion efficiency is obtained in the regime of double layer overlap. In aqueous solutions, this limits optimal channel dimensions to the submicron range. For example, for a $10^{-5} \mathrm{M} \mathrm{KCl}$ aqueous solution a maximum energy conversion efficiency of $\sim 6 \%$ is reached in a channel of $145 \mathrm{~nm}$ height, which is well within our experimental range. Details will be published elsewhere.

In conclusion, we have studied streaming currents in individual silica nanochannels with heights as small as $70 \mathrm{~nm}$ and found that they increase linearly with applied pressure. The streaming current reverses direction when polylysine-coated, positively charged channel walls are used instead of negatively charged fused silica, emphasizing its sensitivity to the polarity of the surface charge. The streaming current increases with channel height, is approximately constant at salt concentrations below $\sim 10 \mathrm{mM} \mathrm{KCl}$, and drops at higher salt. It can be modeled using nonlinear Poisson-Boltzmann theory, combined with a pressure-driven Poiseuille flow in a parallel-plate geometry. A chemical equilibrium model for the surface charge, taken as a boundary condition, predicts streaming currents in nanochannels fairly well over the full range of measured heights and $\mathrm{KCl}$ concentrations, whereas constant surface charge, and in particular constant potential boundary conditions, gave a poor description. This highlights the importance of using appropriate boundary conditions when dealing with electrokinetic phenomena in nanofluidics.

The authors acknowledge discussions with Serge Lemay, and funding from NWO and FOM.
[1] J. Han and H. G. Craighead, Science 288, 1026 (2000).

[2] J. Li et al., Nature (London) 412, 166 (2001).

[3] M. J. Levene et al., Science 299, 682 (2003).

[4] L. R. Huang, E. C. Cox, R. H. Austin, and J.C. Sturm, Science 304, 987 (2004).

[5] J.C.T. Eijkel and A. van den Berg, Microfluidics Nanofluidics 1, 249 (2005).

[6] C. Werner et al., J. Colloid Interface Sci. 208, 329 (1998).

[7] R. B. M. Schasfoort et al., Science 286, 942 (1999).

[8] H. A. Stone, A. D. Stroock, and A. Ajdari, Annu. Rev. Fluid Mech. 36, 381 (2004).

[9] D. Stein, M. Kruithof, and C. Dekker, Phys. Rev. Lett. 93, 035901 (2004).

[10] H. Daiguji, P. Yang, A. J. Szeri, and A. Majumdar, Nano Lett. 4, 2315 (2004).

[11] J. Yang, F. Lu, L. W. Kostiuk, and D. Y. Kwok, J. Micromech. Microeng. 13, 963 (2003).

[12] S. Alkafeef, R. J. Gochin, and A. L. Smith, Colloids Surf. A 195, 77 (2001).

[13] O. El-Gholabzouri, M. A. Cabrerizo, and R. HidalgoÁlvarez, Colloids Surf. A 159, 449 (1999).

[14] L. Renaud, P. Kleimann, and P. Morin, Electrophoresis 25, 123 (2004).

[15] Y. G. Gu and D. Q. Li, J. Colloid Interface Sci. 226, 328 (2000).

[16] S. H. Behrens and D. G. Grier, J. Chem. Phys. 115, 6716 (2001).

[17] R. K. Iler, The Chemistry of Silica (John Wiley And Sons, Inc., New York, 1979).

[18] Reported values for the parameters in the chemical equilibrium model have ranges for $\Gamma=5-8 \mathrm{~nm}^{-2}, p \mathrm{~K}=$ 6-8.5, and $\mathrm{C}=0.2-2.9 \mathrm{Fm}^{-2}$ [R.E. G. van Hal et al., Adv. Colloid Interface Sci. 69, 31 (1996); S. Ong et al., Chem. Phys. Lett. 191, 327 (1992); and Refs. [16,17] ]. $\sigma$ is weakly sensitive to $\Gamma$, and increases with $C$ and $p H-$ $p \mathrm{~K}$. For $p \mathrm{H}-p \mathrm{~K}$ between -0.5 and +1.5 and $C$ between 0.2 and $2.9 \mathrm{~F} / \mathrm{m}^{2}$, we find extremes for $\sigma$ at low salt between -2 and $-8 \mathrm{mC} / \mathrm{m}^{2}$, and at high salt between -15 and $-192 \mathrm{mC} / \mathrm{m}^{2}$. In all calculations we used values of $\eta=0.8904 \mathrm{mPas}$ and $\epsilon=78.54$ for water at $25^{\circ} \mathrm{C}$, at which temperature our experiments were performed. Parameters $\Gamma=8 \mathrm{~nm}^{-2}, p \mathrm{~K}=7.9, p \mathrm{H}=8$, and $C=$ $0.3 \mathrm{Fm}^{-2}$ predict the data best for all channel heights within the reported parameter space.

[19] Parameter values for the solid lines in Fig. 3: $h=$ $70 \mathrm{~nm} \rightarrow p \mathrm{~K}=6.8, \quad C=0.26 \mathrm{Fm}^{-2} ; \quad h=140 \mathrm{~nm} \rightarrow$ $p \mathrm{~K}=7.8, \quad C=0.26 \mathrm{Fm}^{-2} ; \quad h=279 \mathrm{~nm} \rightarrow p \mathrm{~K}=8$, $C=0.2 \mathrm{Fm}^{-2} ; h=563$ and $1147 \mathrm{~nm} \rightarrow p \mathrm{~K}=9, C=$ $0.9 \mathrm{Fm}^{-2} . \Gamma=8 \mathrm{~nm}^{-2}$ and $p \mathrm{H}=8$ are kept constant.

[20] At low salt we observe that the streaming current of the polylysine-coated channel scales nonlinearly with the pressure. We speculate that long polymer chains at the wall may swell at low salt, thereby reducing the effective height, whereas at higher pressure, shear forces at the wall straighten out the polymers by fluid drag, thus increasing the effective height and streaming current. 